\title{
On the Self-Similarity in Biological Processes
}

\author{
Oliver Szasz ${ }^{1}$, Gyula Peter Szigeti², Andras Szasz ${ }^{1}$ \\ ${ }^{1}$ Biotechnics Department, St. Istvan University, Godollo, Hungary \\ ${ }^{2}$ Institute of Human Physiology and Clinical Experimental Research, Semmelweis University, Budapest, Hungary \\ Email: Szasz.Andras@gek.szie.hu
}

How to cite this paper: Szasz, O., Szigeti, G.P. and Szasz, A. (2017) On the SelfSimilarity in Biological Processes. Open Journal of Biophysics, 7, 183-196.

https://doi.org/10.4236/ojbiphy.2017.74014

Received: August 3, 2017

Accepted: September 5, 2017

Published: September 8, 2017

Copyright $\odot 2017$ by authors and Scientific Research Publishing Inc. This work is licensed under the Creative Commons Attribution International License (CC BY 4.0).

http://creativecommons.org/licenses/by/4.0/

\begin{abstract}
We show that the processes described by Avrami functions are self-similar. A comparative function characterizes a self-similar process by a certain Avrami exponent. We define the self-similar categories of some well-known biological processes. The method to determine the Avrami exponent by choosing the comparative function is demonstrated on the diffusion model of the growth of nuclei. We generalize the results.
\end{abstract}

\section{Keywords}

Self-Similar Process, Comparative Function, Avrami Exponent

\section{Introduction}

The self-organizing behavior of the materials is a well-known and widely investigated topic in research [1] and especially in biology [2]. The description of the self-organized, self-controlled (self-limited) processes was firstly in focus in the solid-state reactions (precipitations, phase-transitions, aggregations, nucleation, growth, etc.) pioneering by Kolmogorov [3], Johnson, Mehl [4] and Avrami [5] [6] [7], and later modified by others [8] [9].

The Avrami equation could serve as a mathematical model of different biological processes [10] [11]. Experimental data collected by Cope, [12] [13], and others [14] [15], show a certain universality of the Avrami equation to describe the real processes. The universality makes it possible to study different processes without knowing the exact structure and dynamics of a given system. The situation is similar to the description of the critical phenomena [16], where the physical laws connect to broad, general-function categories.

Our objective in this present work is to derive the universality of the Avramiequation. 


\section{Method}

Self-similarity means the scaling possibility of all the measurable quantities of the studied object. Let us assume that $r$ is the linear size of the scale and $L$ is a scale-dependent property: $L(r)$. By lengthening the scale by $a$ times, the property transforms as

$$
L(a r)=k L(r)
$$

where the factor $k$ depends only on $a$. The power type functional relationship is valid in the biology and the fractal theory:

$$
L(r)=A r^{\alpha}
$$

which changes in the form of

$$
L(a r)=A(a r)^{\alpha}=a^{\alpha} L(r)
$$

When Equation (2) is converted to the log-log scale, the result is a straight line with a gradient equal to the slope.

$$
\lg L(r)=\lg A+\alpha \lg r
$$

The slope usually verifies the self-similarity of fractals.

Definition of the self-similarity in (1) means that the relationship is valid for any $a$ and $k$ independent from $r$. Hence $k$ is the general function of $a$, expressing (1) in the form of

$$
L(a r)=k(a) L(r)
$$

From this, we get a new and more general definition of self-similarity, namely

$$
L: r \in I_{r} \mapsto L(r) \in R^{1}
$$

the mapping is self-similar, if $L(a r)$ may be expressed in a more general form where the scale-transformed function can be separated in the form of a product:

$$
L(a r)=k(a) G(r)
$$

Solving the Equation (7), we suppose that $a=1$. Then,

$$
L(r)=k(1) G(r)=c G(r)
$$

where $c=k(1)$ is a constant. Consequently, $L(r)$ and $G(r)$ differ only in the fixed multiplicator. By the last property the Equation (7) can be rewritten:

$$
L(a r)=\frac{k(a)}{k(1)} L(r)=\eta(a) L(r) \quad\left[\eta(a)=\frac{k(a)}{k(1)}\right]
$$

Repeating the same procedure for the case of $r=1$, the following relationship is valid for any $u, v$ variables:

$$
L(u v r)=\eta(u v) L(r)=\eta(u) \eta(v) L(r)
$$

Therefore, the function $\eta(u v)$ in Equation (10) is also a self-similar function:

$$
\eta(u v)=\eta(u) \eta(v)
$$


The general solution of this function-equation is difficult. We may choose $a=r, r=1$ for the common part of variables from the relationship Equation (9):

$$
L(r)=\frac{k(a)}{k(1)} L(r)=\eta(r) L(1)=C \eta(r)
$$

where $C=L(1)$ is a constant. Consequently, when $L(r)$ is a continuous function on this common set, then it is true also for $\eta(r)$. In this case, the solution of the function Equation (12) is known for positive $r$ values [17]:

$$
\eta(r)=K r^{\alpha}
$$

where $K$ and $\alpha$ are constants. Hence, we get from Equation (12):

$$
L(r)=A r^{\alpha}
$$

where $\mathrm{A}$ is another constant. Consequently, the power-law principle shown in Equation (1), can be applied indeed in general case for the description of selfsimilarity.

The concept of self-similarity may also be extended from the structures to the processes [18]. Let us take a process described by the

$$
f: t \in I=[0, \infty) \mapsto f(t) \in R
$$

function. Let us choose the time $t$ expressed in the form of

$$
t=\tau t^{\prime}
$$

with the aid of an earlier $t^{\prime}$ time. Our statement is that the process described by the function $f$ is self-similar, if

$$
f(t)=f\left(\tau t^{\prime}\right)=g(\tau) h\left(t^{\prime}\right) \quad \forall t, t^{\prime} \in I=[0, \infty)
$$

Since we have proved earlier that the solution of the equation of the above function has the form of

$$
f(t)=a b t^{n}, g(\tau)=a \tau^{n}, \quad h\left(t^{\prime}\right)=b t^{\prime n}
$$

thus, self-similarity can be written in a simpler form of:

$$
f\left(\tau t^{\prime}\right)=f(\tau) f\left(t^{\prime}\right) \quad \forall t, t^{\prime} \in I=[0, \infty)
$$

Equation (19) means that the value of the physical quantity of the process can be established at a later point in time by the extension or shrinking of an earlier value, where the amount of extension or shrinking depends only on the ratio of the two time points. Therefore, the ratio of function values is the function of the ratio of time points. A solution of Equation (19) (Cauchy-type function-equation) is [17]:

$$
f(t)=t^{\alpha}
$$

where $\alpha$ is an arbitrary number. Of course, in nature, not every process is selfsimilar. Those deserve attention, which can be transformed them into self-similar processes. When $f$ is not self-similar, we may apply a suitably chosen $u$ transformation, when the composition of $u \circ f$ will be self-similar, namely: 


$$
u\left[f\left(\tau t^{\prime}\right)\right]=g(\tau) h\left(t^{\prime}\right) \quad \forall t, t^{\prime} \in I=[0, \infty)
$$

The $u$ function is named: comparative function. It is proven [19] [20], that the comparison functions of the most common processes scarcely differ from each other when the appropriate parameters are chosen.

A wide group of processes differ regarding mathematics; however, a process can be transformed into self-similar one with the help of a comparative function scarcely dissimilar in approximation capability.

We are going to show that the power type equation is the consequence of some simple and very general assumptions.

\section{Results}

The Avrami equation [5] [6] [7] has the following form:

$$
x(t)=1-\mathrm{e}^{-(c t)^{\alpha}}
$$

where $t$ is the time of the reference system, and $\alpha$ and $c$ are constants. (The function is identical to the well-known Weibull function [21], which is widely used to describe the reliability/aging processes [22].) Let us rewrite Equation (22) into another form:

$$
g(t)=\ln \frac{1}{1-x}=(c t)^{\alpha}
$$

Moreover, it is transformed to a new time-scale by the

$$
t \mapsto v=c t
$$

linear transformation. Following these changes, the Equation (22) Avrami-equation takes its final form as:

$$
f(v)=\ln \frac{1}{1-x}=(v)^{\alpha}
$$

The function $\ln \frac{1}{1-x}$ is the comparative function for the Avrami processes, (Avrami comparative function). Consequently, the Equation (24) linear transformation could be chosen when the Equation (25) Avrami comparative function is self-similar.

In the case when $\mathrm{f}$ is a continuously differentiable function, the function Equation (19) is identical to the following differential equation:

$$
\frac{\mathrm{d} f}{\mathrm{~d} v}=\alpha \frac{f}{v}
$$

Substituting function Equation (25) into Equation (26), we get the differential equation:

$$
\frac{\mathrm{d} x}{\mathrm{~d} v}=\alpha v^{\alpha-1}(1-x)
$$

Transforming Equation (27) back to the reference time-scale, we obtain the differential equation describing the dynamic features of the process.

$$
\frac{\mathrm{d} x}{\mathrm{~d} t}=c \alpha(c t)^{\alpha-1}(1-x)
$$


It is proven [19] [20] that the comparison functions of the most common processes scarcely differ from each other when the appropriate parameters are chosen (Figure 1).

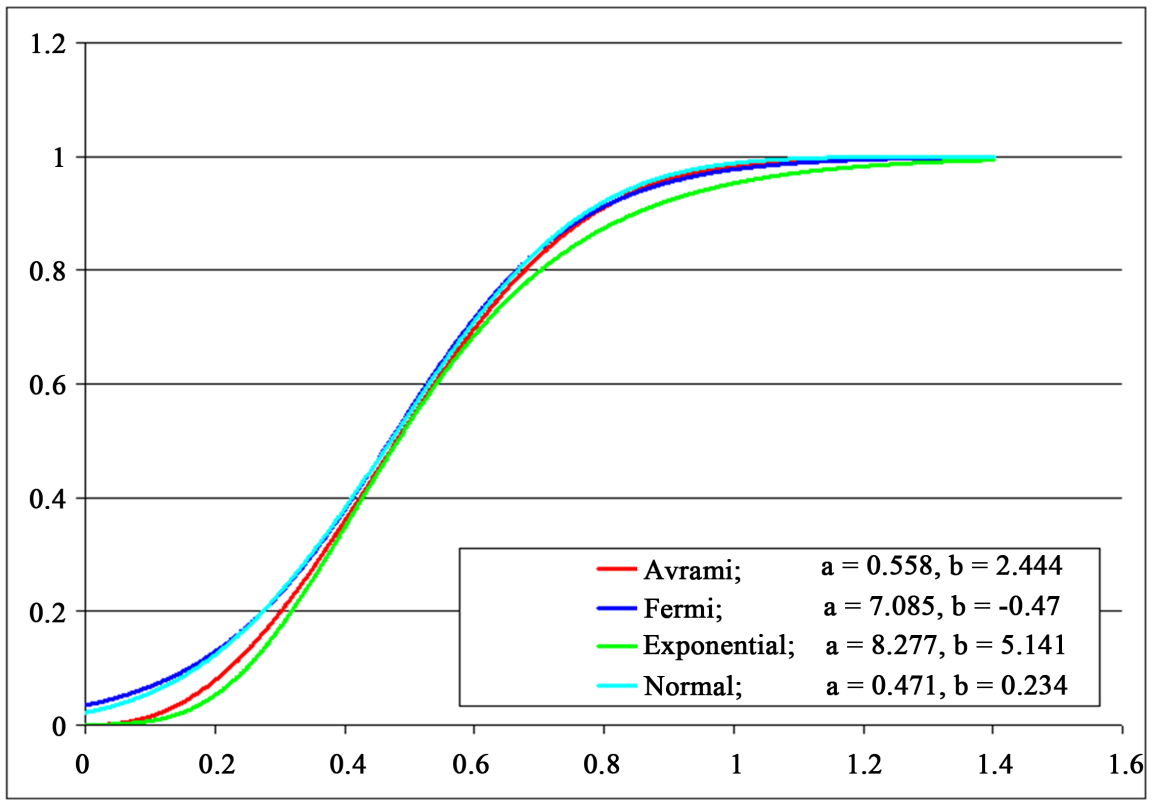

(a)

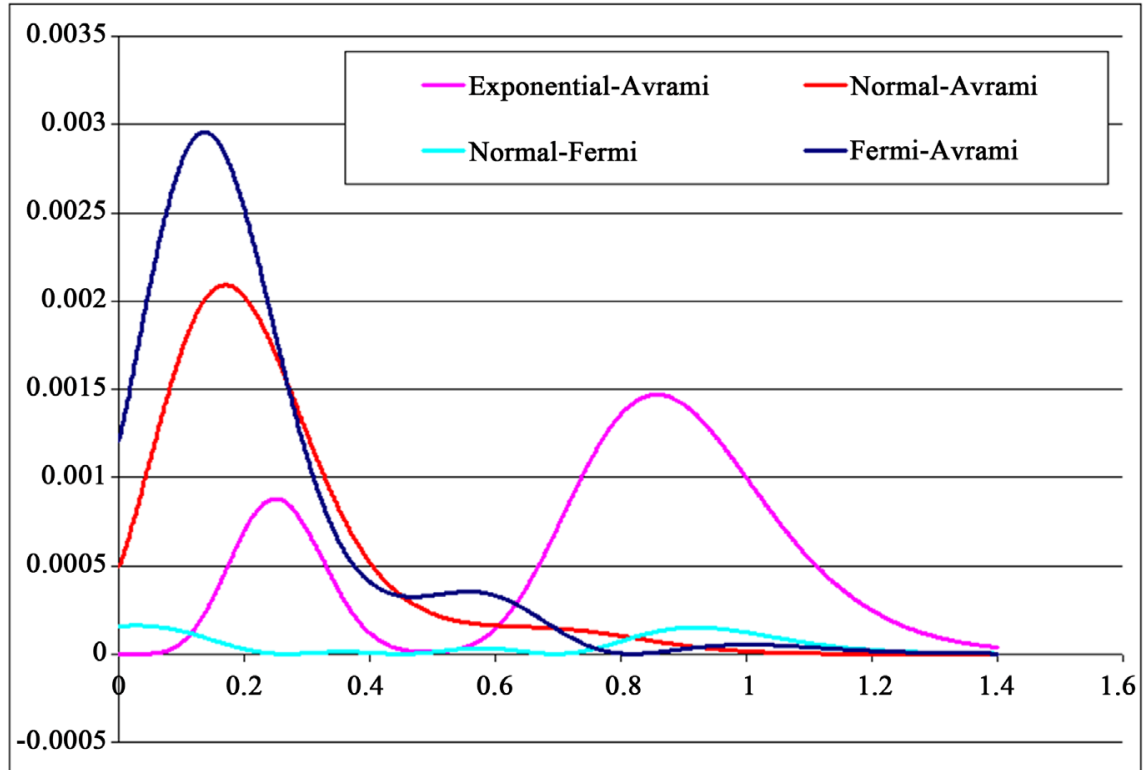

(b)

Figure 1. A particular set of parameters allows the transformed functions to be identical within 0.3\% accuracy. $\left(\mathrm{R}^{2}\right.$ Avrami-Fermi $=0.99981, \mathrm{R}^{2}$ Avrami-Exponent. $=0.99915, \mathrm{R}^{2}$ Avrami-Normal $=0.99986, \mathrm{R}^{2}$ Exponent.-Fermi $=0.99894, \mathrm{R}^{2}$ Exponent.-Normal $=$ $0.99846, \mathrm{R}^{2}$ Normal-Fermi $\left.=0.99984\right)$. Upper panel: the given transformed functions, lower panel: the square of their deviation. Fermi $:=\frac{1}{\exp (-a(x+b))+1}$;

Exponential $:=\exp (-a \exp (-b x)) ;$ Avrami $:=\exp \left(-a x^{b}\right)$. 
A wide group of processes differs regarding mathematics. However, it can be transformed into a self-similar one with the help of a comparative function hardly dissimilar in approximation capability.

\section{Discussion}

With our results, we could explain numerous important processes in biology. Let us describe first the growth of nuclei in a homogeneous thermodynamic system. For the sake of simplicity, consider the nuclei as a sphere, and the inside solid material has a constant number of particles by volume units $n_{0}$, as well as the concentration distribution of the particles in the neighborhood of the nuclei considered as having spherical symmetry. For the diffusion growth of these nuclei, the conservation of the particles could be formulated:

$$
\frac{\mathrm{d} N}{\mathrm{~d} t}=I
$$

where $N$ is the number of the particles in the nuclei, $I$ is the particle current on the surface of the nuclei. If the nuclei radius is $r$ and it is homogenous, then

$$
N=n_{0} \frac{4 \pi}{3} r^{3}
$$

moreover, the particle current

$$
I=4 \pi r r^{2} j
$$

where $j$ is the current density of particles. If $j=$ const. then

$$
r=\frac{j}{n_{0}} t
$$

The exponent, in this case, is trivially $n=1$. Do not forget: this is a particular instance when both the concentration gradient and the thickness of the boundary of the nuclei are constant. Let us study when only the current density kept constant on the nuclei boundary. Then, based on (25):

$$
n_{0} 4 \pi r^{2} \frac{\mathrm{d} r}{\mathrm{~d} t}=I=4 \pi r^{2} D \frac{\partial n}{\partial r}=\text { all. }
$$

where $D$ is the diffusion constant. If the concentration distribution is stationary, then

$$
n_{0} 4 \pi r^{2} \frac{\mathrm{d} r}{\mathrm{~d} t}=I=4 \pi \operatorname{Dr}\left[n_{\infty}-n(r)\right]
$$

where $n_{\infty}$ is the particle concentration far from the nuclei. The particle concentration of the nuclei could be written [23]:

$$
n(r)=n_{\text {plane }}\left(1+\frac{2 \alpha}{n_{0} k T r}\right)
$$

where $n_{\text {plane }}$ is the concentration at a plane, $\alpha$ is the surface tension, and $T$ is the temperature. If $\left(\alpha / n_{0}\right) \ll 1$, then 


$$
\frac{2 \alpha}{n_{0} k T r} \ll 1
$$

Hence from the condition Equation (36) and Equation (35):

$$
r \approx t^{\frac{1}{2}}
$$

so the exponent is $n=0.5$. Contrarily, if $\left(\alpha / n_{0}\right) \gg 1$, then

$$
r \approx t^{\frac{1}{3}}
$$

so the exponent is $n=1 / 3$. Consequently, the volume of the nuclei depends on the following:

$$
V_{\text {grain }} \approx t^{3 n}
$$

which is the Avrami exponent.

Let us calculate the Avrami comparative function for the same conditions. Denote the part of the old phase transforming into the new one by $x$. According to the Kolmogorov-Avrami assumptions [3] [5] [6] [7], the speed of the phase transformation is proportional to the growth rate of the nuclei volume ( $\left.\mathrm{d} V_{\text {nucl }} / \mathrm{d} t\right)$ and the non-transformed part of the phase:

$$
\frac{\mathrm{d} x}{\mathrm{~d} t} \approx \frac{\mathrm{d} V_{\text {nucl }}}{\mathrm{d} t}(1-x)
$$

Hence, by integration of (40), the $V_{\text {nuci }}$ :

$$
\ln \frac{1}{1-x} \approx V_{\text {nucl }}
$$

Using the $k=$ const. factor, we get:

$$
\ln \frac{1}{1-x}=k t^{3 n}
$$

Consequently, the nuclei growth process by diffusion with the

$$
\ln \frac{1}{1-x}
$$

Avrami comparative function and on the

$$
k^{\frac{1}{3 n}} t
$$

time-scale is a self-similar process and could be described by the Avrami exponent:

$$
\alpha=3 n
$$

A summary of these approximations is shown in Table 1.

To generalize the above, let us denote a cluster size with arbitrary shape by $\xi$. In this case, we can assume its volume is $V_{\text {nucl }} \approx \xi^{k}$. Hence the Avrami-exponent would be

$$
\alpha=\beta n
$$

The actual value of $\beta$ depends on the form of the cluster, e.g. in the needle 
Table 1. Avrami exponent at growth of nuclei by diffusion.

\begin{tabular}{cc}
\hline Character of the process & Avrami exponent $(\alpha)$ \\
\hline Constant-current density of the diffusion & 3 \\
Constant flux of the diffusion, with $\left(\alpha / n_{0}\right) \ll 1$ & $3 / 2$ \\
Constant flux of the diffusion, with $\left(\alpha / n_{0}\right) \gg 1$ & 1 \\
\hline
\end{tabular}

form; it is $\beta=1$; in the planar form, $\beta=2$. The above considerations could be extended even for fractal dimensions: $\beta=\mathrm{dfr}$, where $\mathrm{dfr}$ is the measured fractal dimension.

Various processes of class $\alpha=1.0$ are summarized in Table 2 to refer to the time-scale $v=c t$.

The process character is the Avrami exponent $\alpha$. Based on the extensive works of FW Cope [10] [11], we had collected the Avrami exponents for various solidstates (Table 3 ) and biological processes (Table 4).

The method could also be generalized on vectorial processes. Assuming that the processes are disjointed and the individual modes have the same comparative functions, then all the $g_{i}(t)$ comparative functions of the modes could be transformed into the form:

$$
g_{i}(t)=\left(c_{i} t\right)^{\alpha},(i=1,2, \cdots, N)
$$

where $c_{i}$ is the scale factor of the time-scale of the $\mathrm{i}$-th mode and $N$ is the number of modes. The example to study could be a bioprocess, chemical catabolism, exchanging materials with the actual neighborhood. There could be two reasons to change the number of particles in the system: a breakdown of a molecule or a molecular exchange at the surface of the system. We must consider not only one but numerous chemical components participating in this process. It could happen even in the simplest double-component system, not only because of the chemical potential of the breaking but also because the final component differs from its environmental values. Under these circumstances, the diffusion of both compounds starts and becomes coupled according to Onsager's non-equilibrium [25]. The particle numbers of the components ( 1 and 2 denote the original and the decomposed components, respectively) could be described by:

$$
\begin{aligned}
& \frac{\mathrm{d} x_{1}}{\mathrm{~d} t}=J_{k 1}-\lambda x_{1} \\
& \frac{\mathrm{d} x_{2}}{\mathrm{~d} t}=J_{k 2}+\lambda x_{1}
\end{aligned}
$$

where $\lambda$ is the constant of the decomposition. On the other hand, for $J_{k 1}$ and $J_{k 2}$, the Onsager equations determine particle currents:

$$
\begin{aligned}
& J_{k 1}=-D_{11} x_{1}-D_{12} x_{2} \\
& J_{k 2}=-D_{21} x_{1}-D_{22} x_{2}
\end{aligned}
$$

where the matrix of $D_{11}, D_{12}, D_{21}$ and $D_{22}$ conduction constants is a positive definite structure due to the second (entropy) law of thermodynamics. Furthermore, 
Table 2. Processes with $\alpha=1.0$ (time-scale $v=c t$ ).

\begin{tabular}{ccc}
\hline Process & Descriptive function & Comparative function \\
\hline Decomposition, breakdown & $x=x_{\infty} \mathrm{e}^{-c t}$ & $\ln \frac{x_{\infty}}{x}$ \\
Infinite proliferation & $x=x_{\infty} \mathrm{e}^{c t}$ & $-\ln \frac{x_{\infty}}{x}$ \\
Logistic process by Fermi distribution & $x=1-\frac{x_{\infty}}{1+\mathrm{e}^{-c t}}$ & $\ln \frac{1-x}{x_{\infty}+x-1}$ \\
Logistic process by Gompertz distribution & $x=x_{1 \infty} \mathrm{e}^{-x_{2 \infty} \mathrm{e}^{-\alpha t}}$ & $\ln \frac{x_{2 \infty}}{\ln \frac{x_{1 \infty}}{x}}$ \\
Demographic explosion & $x=\frac{x_{\infty}}{\pi} \operatorname{arccot}(c t)$ & $\cot \frac{x}{x_{\infty}} \pi$ \\
\hline
\end{tabular}

Table 3. Avrami exponents of some solid-state processes [24].

\begin{tabular}{cc}
\hline Processes in solids [24] & Avrami exponent $(\alpha)$ \\
Beginning of nucleation & 3 \\
Nucleation with constant speed & 4 \\
Nucleation with growing speed & $<4$ \\
Staring nucleation and surface growth & 2 \\
Diffusional growth from nuclei & 1.5 \\
Diffusional growth from growing nuclei & 2.5 \\
Diffusional growth in 1 or 2 dimensions & 1 \\
Limited diffusional growth in 1 or 2 dimensions & 1.5 \\
\hline
\end{tabular}

Table 4. Avrami exponents of some biological processes [19].

\begin{tabular}{cc}
\hline Biological processes [10], [11] & Avrami exponent $(\boldsymbol{\alpha})$ \\
\hline Growth of bacteria & 1.9 \\
K+ conductance decay in nerve axon & 1.7 \\
K+ leakage from poisoned muscle & 2.0 \\
Growth (weight) of rat & 2.3 \\
Growth (length) of regular leg of salamander & 1.9 \\
Growth (height) of sunflower plant & 2.2 \\
Synthesis of chlorophyll in maize plant & 1.21 \\
Muscle tension during tetanic contraction & 1.24 \\
Myosin splitting of ATP & 1.0 \\
Fresh green leaf IR phosphorescence decay & 1.28 \\
Dried green leaf IR phosphorescence decay & 1.08 \\
Melanin IR phosphorescence decay & 1.16 \\
Cytochrome c IR phosphorescence decay &
\end{tabular}


the set of the equation is symmetrical $\left(D_{12}=D_{21}\right)$ according to the Onsager's theory, [25]. Combining Equation (48) and Equation (49), we get the following vectorial differential equation:

$$
\frac{\mathrm{d}}{\mathrm{d} t}\left[\begin{array}{l}
x_{1} \\
x_{2}
\end{array}\right]+\left[\begin{array}{cc}
D_{11}+\dot{\lambda} & D_{12} \\
D_{21}-\dot{\lambda} & D_{22}
\end{array}\right] \cdot\left[\begin{array}{l}
x_{1} \\
x_{2}
\end{array}\right]=\left[\begin{array}{l}
0 \\
0
\end{array}\right]
$$

describing the time variation of the particle currents. The modes correspond with the eigenvalues of the coefficient matrix. The time dependence of the modes:

$$
\mathrm{e}^{\eta t}
$$

moreover, the characteristic equation to determine the eigenvalues:

$$
\operatorname{det}\left[\begin{array}{cc}
D_{11}+\lambda-\eta & D_{12} \\
D_{21}-\lambda & D_{22}-\eta
\end{array}\right]=0
$$

Hence, we get:

$$
\eta_{1,2}=\frac{-\left(D_{11}-D_{22}+\lambda\right) \pm \sqrt{\left(D_{22}-D_{11}-\lambda\right)^{2}+4 D_{12}^{2}-4 D_{12} \lambda}}{2}
$$

Both the modes of the vectorial process belong to the class $\alpha=1.0$, with the $\ln \frac{x_{i \infty}}{x_{i}},(i=1,2)$ comparative function and with $v_{1}=\eta_{1} t$ and $v_{2}=\eta_{2} t$ time scale.

Description of the self-similar traveling waves is also possible in above format. Let us denote the variable for the traveling wave:

$$
\xi:=v t+y
$$

which belongs to a wave traveling in the y-direction at velocity v. According to our previous results, the comparative function is:

$$
g(y, t)=(c \xi)^{\alpha}
$$

The comparative functions are similar by an acceptable accuracy with appropriately chosen parameters. Let us choose the Avrami-like comparative function. By this, the traveling wave could be described by the following wave function:

$$
x(y, t)=1-\mathrm{e}^{-[c(v t+y)]^{\alpha}}
$$

This wave is not a solution because the corresponding

$$
\frac{\mathrm{d} x}{\mathrm{~d} \xi}=c \alpha(c \xi)^{\alpha-1}(1-x)
$$

Avrami-equation leads to a linear equation with non-constant coefficients:

$$
\frac{1}{v} \frac{\partial x}{\partial t}+\frac{\partial x}{\partial y}-2 c \alpha(c(v t+y))^{\alpha-1}(1-x)=0
$$

The two-component autocatalytic reactions could be described by the following vector equation [26] [27] [28]: 


$$
\begin{aligned}
& \frac{\mathrm{d} x_{1}}{\mathrm{~d} v}=x_{1}\left(1-x_{2}\right) \\
& \frac{\mathrm{d} x_{2}}{\mathrm{~d} v}=-x_{2}\left(1-x_{1}\right)
\end{aligned}
$$

Introducing the variable of the traveling wave by Equation (54) and regarding the process as self-similar by Equation (55), then we receive the following coupled vectorial wave-equations:

$$
\begin{aligned}
& \left(\frac{\partial}{\partial t}+v_{1} \frac{\partial}{\partial y}\right) x_{1}=x_{1}\left(1-x_{2}\right) \\
& \left(\frac{\partial}{\partial t}+v_{2} \frac{\partial}{\partial y}\right) x_{2}=-x_{2}\left(1-x_{1}\right)
\end{aligned}
$$

A solution of Equation (60) was made with bilinear transformation by Hirota [29] and with Malfliet [30], obtaining a shock-wave:

$$
x_{1}=x_{2}=\frac{1}{2}\left\{1+\tanh \left[\frac{v_{1}+v_{2}}{2}\left(y+\left(v_{1}-v_{2}\right) t\right)\right]\right\}
$$

This equation is very similar to the various assumptions (e.g. [31] [32] [33] [34] [35]) of the energy-pack-like information transfer. Consequently, these biological processes presumably could be described by such self-similar function classes, based on the dynamical observations for the long-range correlation lengths ([36] [37] [38]). The long-range correlation could be derived from self-similar time structures establishing a new discipline (Fractal Physiology, [39] [40] [41] [42]). The fundamental role of the self-similarity in biology is well recognized in the scaling behavior of living objects [43] [44] [45].

\section{Conclusion}

In this paper, we show the possible transformation of the mathematical models of biological processes to the self-similar processes with the help of an appropriate comparative function. The mathematical expressions of the applied comparative functions are different, but by the suitable choice of their parameters, they approach each other well with little error. Consequently, in the practical solutions, to use one of them for the various tasks is satisfactory. The Avrami equation will describe the mathematical model of the processes with Avrami-like comparative function. We believe this is the reason for the unusual universality of the Avrami equation in biology. Consequently, the self-organizing conditions and the analogy of the self-organized processes could be a fruitful heuristic method in biological model calculations.

\section{References}

[1] Wolfram, S. (2002) A New Kind of Science. Wolfram Media Inc.

[2] Camazine, S., Deneubourg, J.-L., Franks, N.R., Sneyd, J., Theraulaz, G. and Bonabeau, E. (2003) Self-Organization in Biological Systems. Princeton Studies in Complexity, Princeton Univ. Press, Princeton-Oxford.

[3] Kolmogorov, N.N. (1937) On the Statistical Theory of the Crystallization of Metals. 
Bull. Acad. Sci. UssR, Math. Ser., 1, 355.

[4] Joshnson, W.A. and Mehl, P.A. (1939) Reaction Kinetics in Processes of Nucleation and Growth. Trans. Amer. Inst. Mining (Metal) Engrs, 135, 416.

[5] Avrami, M.A. (1939) Kinetics of Phase Change. Parts: I-II-III. The Journal of Chemical Physics, 7, 1103.

[6] Avrami, M.A. (1940) Kinetics of Phase Change. Parts: I-II-III. The Journal of Chemical Physics, 8, 212.

[7] Avrami, M.A. (1941) Kinetics of Phase Change. Parts: I-II-III. The Journal of Chemical Physics, 9, 117.

[8] Augis, J.A. and Bennett, J.E. (1978) Calculation of the Avrami Parameters for Heterogeneous Solid-State Reactions Using a Modification of the Kissinger Method. Journal of Thermal Analysis and Calorimetry, 13, 285-291. https://doi.org/10.1007/BF01912301

[9] Levine, L.E., Lakshimi, N-K. and Kelton, K.F. (1997) Finite Size Corrections for the John-Son-Mehl-Avrami-Kolmogorov Equation. Journal of Materials Research, 12, 124-131. https://doi.org/10.1557/JMR.1997.0020

[10] Cope, F.W. (1977) Detection of Phase Transitions and Cooperative Interactions by Avrami Analysis of Sigmoid Biological Time Curves for Muscle, Nerve, Growth, Firefly, and Infrared Phosphorescence, of Green Leaves, Melanin and Cytochrome C. Physiological Chemistry and Physics, 9, 443-459.

[11] Cope, F.W. (1977) Solid State Physical Replacement of Hodgkin-Huxley Theory. Phase Transformation Kinetics of Axonal Potassium Conductance. Physiological Chemistry and Physics, 9, 155-160.

[12] Cope, F.W. (1977) The Kinetics of Biological Phase Transitions Manifested by Sigmoid Time Curves: A Review of Approaches. Physiological Chemistry and Physics, 8, 519-527.

[13] Cope, F.W. (1980) Avrami Analysis of Electrical Switching in Hydrated Melanin Suggest Dependence on a Phase Transition. Physiological Chemistry and Physics, 12, 537-538.

[14] Suckjoon, J. and Bechhoefer, J. (2005) Nucleation and Growth in One Dimension, II. Applicationto DNA Replication Kinetics. Physical Review E, 71, Article ID: 011909.

[15] Zhang, H. (2005) Reconstructing DNA Replication Kinetics from Small DNA Fragments. Ms. Thesis, Simon Fraser University.

[16] Binney, J.J., Dowrick, N.J., Fisher, A.J. and Newman, M.E.J. (1992) The Theory of Critical Phenomena: An Introduction to the Renormalization Group. Oxford Clarendon Press.

[17] Aczél, J. (1961) Functional Analysis and Its Applications. VEB Deutsche Verlag der Wissenschaften, Berlin. (In German)

[18] Emrechts, P. and Maejima, M. (2002) Self-Similar Processes, Princeton Series in Applied Mathematics. Princeton University Press, Princeton Oxford.

[19] Mar'yan, M.I. and Szasz, A. (2016) Self-Organizing Processes Is Non-Crystalline Materials: From Lifeless to Living Objects. Oncothermia Journal, 16, 8-167.

[20] Wilson, D.L. (1994) The Analysis of Survival (Mortality), Data: Fitting Gompertz, Weibull and Logistic Functions. Mechanisms of Ageing and Development, 74, 15-33.

[21] Weibull, W. (1951) A Statistical Distribution Function of Wide Applicability. Journal of Applied Mathematics, 18, 293-297. 
[22] Meeker, W.Q. and Escobar, L.A. (1998) Statistical Methods for Reliability Data. John Wiley \& Sons, Inc., New York.

[23] Frenkel, J.I. (1957) Statistical Physics. Akademie Verlag, Berlin. (In German)

[24] Guy, A.G. (1972) Introduction to Material Science. McGraw Hill Book Company, New York.

[25] Katchalsky, A. and Curran, P. (1967) Nonequilibrium Thermodynamics in Biophysics. Harvard University Press, Cambridge.

[26] Haken, H. (1978) Synergetics: An Introduction. Springer-Verlag, Berlin Heidelberg. https://doi.org/10.1007/978-3-642-96469-5

[27] Volterra, V. (1931) Lectures of Mathematical Theory of Life. Paris. (In French)

[28] Lotka, A.J. (1956) Elements of Mathematical Biology. New York.

[29] Dold, A. and Eckmann, B. (1974) Backlund Transformations. Lecture Notes in Mathematics, Vol. 515, Spinger-Verlag, Berlin.

[30] Malfliet, W. (1992) Solitary Wave Solutions of Nonlinear Wave Equations. American Journal of Physics, 60, 650-654. https://doi.org/10.1119/1.17120

[31] Davidov, A.S. (1978) Solitons Bioenergetics and Mechanism of Muscle Contraction. International Journal of Quantum Chemistry, 16, 5-17. https://doi.org/10.1002/qua.560160104

[32] Davidov, A.S. (1982) Biology and Quantum Mechanics. Pergamon Press, Oxford.

[33] Del Giudice, E., Doglia, S. and Milani, M. (1983) Self-Focusing and Ponderomotive Forces of Coherent Electric Waves: A Mechnaism of Cytoscaleton Formation and Dynamics. In: Frolich, H. and Kremer, F., Eds., Coherent Excitations in Biological Systems, Springer Verlag, Berlin, 123-127. https://doi.org/10.1007/978-3-642-69186-7_12

[34] Szasz, A. (1991) An Electronically Driven Instability: The Living State (Does the Room-Temperature Superconductivity Exist?). Physiological Chemistry and Physics, 23, 43-50.

[35] Szasz, A., vanNoort, D., Scheller, A. and Douwes, F. (1994) Water States in Living Systems. I. Structural Aspects. Physiological Chemistry and Physics and Medical $N M R, 26,299-322$.

[36] Frolich, H. (1988) Biological Coherence and Response to External Stimuli. Springer Verlag, Berlin Heidelberg. https://doi.org/10.1007/978-3-642-73309-3

[37] Szendro, P., Vincze, G. and Szasz, A. (2001) Pink Noise Behaviour of the Bio-Systems. European Biophysics Journal, 30, 227-231.

https://doi.org/10.1007/s002490100143

[38] Voss, R.F. (1989) Random Fractals: Self-Affinity in Noise, Music, Mountains and Clouds. Physica D, 38,362-371.

[39] West, B.J. (1990) Fractal Physiology and Chaos in Medicine. World Scientific, Singapore, London. https://doi.org/10.1142/1025

[40] Bassingthwaighte, J.B., Leibovitch, L.S. and West, B.J. (1994) Fractal Physiology. Oxford University Press, New York, Oxford. https://doi.org/10.1007/978-1-4614-7572-9

[41] Walleczek, J. (2000) Self-Organized Biological Dynamics \& Nonlinear Control. Cambridge University Press, Cambridge. https://doi.org/10.1017/CBO9780511535338

[42] Musha, T. and Sawada, Y. (1994) Physics of the Living State. IOS Press, Amsterdam.

[43] West, G.B., Brown, J.H. and Enquist, B.J. (1999) The Four Dimension of Life: Frac- 
tal Geometry and Allometric Scaling of Organisms. Science, 284, 1677-1679.

[44] Brown, J.H. and West, G.B. (2000) Scaling in Biology, Santa Fe Institute Studies in the Sciences of Complexity. Oxford University Press, Oxford.

[45] Calder, W.A. (1996) Size, Function and Life History. Dover Publications, Inc., Mineola, New York. 